\title{
CONSUMO Y CREACIÓn AUdiovisual EN EUSKera de LOS ADOLESCENTES GIPUZKOANOS: SOBREVIVIR EN UN CONTEXTO Digital DOMinado POR LENGUAS HEGEMÓNICAS
}

\section{AUDIOVISUAL CONSUMPTION AND CREATION IN THE BASQUE LANGUAGE BY YOUNG PEOPLE IN GIPUZKOA: SURVIVING IN A DIGITAL CONTEXT DOMINATED BY HEGEMONIC LANGUAGES}

\author{
Ainhoa Fernandez de Arroyabe, Iñaki Lazkano, y Leire Eguskiza \\ Facultad de Ciencias Sociales y de la Comunicación, Universidad del País Vasco, España \\ *autora de contacto: ainhoa.fernandezdearroyabe@ehu.eus
}

\begin{abstract}
Resumen: El presente artículo examina el consumo y la creación de contenidos audiovisuales en euskera de los adolescentes gipuzkcoanos. Analiza qué consumen y crean, qué clase de dispositivos utilizan, y en qué medida influye en su elección la Red, un ecosistema digital dominado por las lenguas hegemónicas. Este escrito se asienta sobre una amplia investigación que implicó a 2.642 alumnos de la ESO (12-16 años) de los modelos lingǘsticos B y D de 60 centros de Gipuzkoa, y concluye que el consumo audiovisual se realiza, fundamentalmente, en castellano. No obstante, se constata la existencia de una brecha entre las comarcas en lo que respecta al consumo en euskera. Asimismo, de esta investigación se infiere, entre otras cosas, que el smartphone es la herramienta esencial del consumo adolescente, que existen diferencias de género tanto en el consumo como en la creación de contenidos, y que los adolescentes son más consumidores que creadores.
\end{abstract}

Palabras clave: Consumo audiovisual, adolescentes, euskera, creación audiovisual, smartphone, Internet

\begin{abstract}
This article examines the audiovisual content consumption and creation in Basque among adolescents of Gipuzkoa. It has been analyzed what do they consume and create, which type of electronic devices do they use, and the extent to which the Net influences their choice, in a digital ecosystem dominated by hegemonic languages. The work is based on a wide investigation in which 2.642 students of secondary education (12-16 years old), belonging to B and D language models in 60 schools, got involved. The results show that Spanish is the main language used for audiovisual consumption. Nevertheless, it can be confirmed the existence of a gap among the different regions of Gipuzkoa related to this consumption in Basque. The study also indicates that smartphones are essential tools for adolescents, that there are gender differences in both the consumption and the content creation, and that teenagers are more consumers than creators.
\end{abstract}

Key words: Audiovisual consumption, adolescents, Basque, audiovisual creation, smartphone, Internet.

EUROPEAN PUBLIC \& SOCIAL INNOVATION
European Public \& Social Innovation Review

Volume 3, Issue 1, 2018 ISSN 2529-9824

Creative Commons 4.0. International 


\section{Introducción}

El objetivo del estudio es identificar el uso del euskera entre los jóvenes guipuzcoanos de 12 a 16 años a la hora de consumir, crear y difundir contenidos audiovisuales en Internet. En tanto que lengua no hegemónica, el euskera se enfrenta al reto de competir por la audiencia tanto de los contenidos audiovisuales tradicionales como de los nuevos formatos digitales que se producen en lenguas hegemónicas como el castellano, pero también, aunque en menor medida, en inglés. Esta batalla se libra en dos ámbitos: el campo de los medios de comunicación tradicionales -ya sean generalistas, temáticos o locales- cuya oferta se ha atomizado al máximo, y en Internet. En este sentido, los adolescentes son un público objetivo crucial porque sus hábitos de consumo actuales condicionan sus preferencias futuras y, sobre todo, porque su rol en el entorno comunicativo contemporáneo ha cambiado de manera radical. El acceso a las nuevas tecnologías, el uso de las redes sociales y de los smartphones ha propiciado que el espectador pasivo del pasado se convierta, si así lo desea, en un prosumidor; es decir, en un consumidor que es a su vez creador de contenidos que difunde y comparte entre sus pares.

Los resultados de esta investigación pretenden obtener una radiografía fidedigna sobre el tipo de contenidos que consumen y crean los adolescentes guipuzcoanos, en qué lengua lo hacen y cómo los comparten en Internet. Este diagnóstico facilita el diseño de políticas públicas eficaces que impulsen el uso del euskera entre los jóvenes a la hora de consumir en este sentido $\mathrm{Y}$ también servirá de fuente de información para los medios tradicionales, ya que recoge los datos de la audiencia adolescente de sus programas.

Por todo ello, la investigación plantea las siguientes Research Questions:

- RQ1. ¿En qué lenguas consumen contenidos los jóvenes?

- RQ2. ¿Qué clase de consumo audiovisual realizan los jóvenes guipuzcoanos?

- RQ3. ¿Existen diferencias en el consumo y creación de contenidos a nivel comarcal?

- RQ4. ¿Cuáles son las principales herramientas que utilizan los jóvenes?

- RQ5. ¿Qué tipo de contenidos crean los adolescentes y en qué idiomas lo hacen?

\section{Marco teórico}

\subsection{Usos y hábitos de consumo y creación de la Generación $Z$}

Los adolescentes del nuevo milenio, jóvenes que han nacido en el seno de la Generación Z (Howe \& Strauss, 2000), se integran en el actual ecosistema digital. Las nuevas tecnologías, las redes sociales y los dispositivos móviles son parte de su universo; un mundo virtual que transforma radicalmente sus relaciones sociales, patrones de consumo y valores. Los adolescentes de la Generación Z tienen unos rasgos característicos: son expertos en la comprensión tecnológica, abiertos socialmente desde las tecnologías, rápidos e impacientes, interactivos, multitarea, y resilientes (FernándezCruz \& Fernández-Díaz, 2016).

En el marco de esa "Sociedad Red" (Castells, 2006), con la cultura participativa y la sociabilidad como referentes primordiales (Aranda, Sánchez Tabernero, \& Tubella, 2010), Internet se convierte en la herramienta que satisface sus necesidades informativas, educativas y de ocio, que pivotan alrededor del entretenimiento y las relaciones interpersonales (Buckingham \& Martínez, 2013).

La personalización, el consumo a demanda y la multiplicidad de pantallas constituyen la base del nuevo ocio adolescente. Un modelo de ocio juvenil "conectado, móvil, transmedia y multisoporte" (Viñals, 2016: 99), que ha adquirido "unos rasgos basados en el cambio constante, la aceleración, el disfrute breve de las cosas, la transitoriedad, la fragilidad de los vínculos humanos y la incertidumbre constante" (Viñals, 2016: 102).

No obstante, aunque la red ofrece múltiples oportunidades de entretenimiento y de interrelación personal, implica también riesgos evidentes. Sobre todo, en el caso específico de los adolescentes, un segmento social muy vulnerable. $\mathrm{La}$ adicción a Internet, el ciberacoso o la sobreexposición son algunas de las principales amenazas sobre las que advierten las diversas investigaciones académicas que analizan los peligros de la Red (Gabelas \& Lazo, 2008; Livingstone \& Helsper, 2010; Catalina, López de Ayala, \& García, 2014; Durán \& Martínez-Pecino, 2015; Montes, García \& Menor, 2018). 


\subsection{El smartphone, redes sociales $y$ consumo multipantalla}

Los adolescentes se diferencian de las generaciones precedentes porque "han modificado sus dietas mediáticas y cada vez navegan más por Internet en detrimento del consumo de televisión" (Fernández Planells \& Figueras-Maz, 2014: 177). Estos jóvenes conforman un público diferenciado que se caracteriza por sus usos $\mathrm{y}$ hábitos exclusivos. A pesar de que la televisión mantiene todavía una importancia significativa (Gewerc, Fraga \& Rodes, 2017), los jóvenes comienzan a abandonar paulatinamente la pantalla televisiva por nuevos espacios que les garanticen un visionado multipantalla y multitarea.

El visionado adolescente se traslada, en gran medida, a las redes sociales, donde irrumpe la "televisión social" y los jóvenes "interactúan entre sí y con los emisores intercambiando opiniones escritas y mensajes audiovisuales" (Teso \& Piñuel, 2014: 95). No obstante, la modificación de la dieta mediática no ha supuesto una exclusión de los medios convencionales, sino, más bien, una diversificación de roles (Fernández-Planells \& Figueras-Maz, 2012; Marín, Yaguana \& Barrazueta. 2013). Por tanto, mientras la televisión se utiliza como una forma de desconexión, Internet se vincula con el entretenimiento y la socialización. Así las cosas, el televisor se adscribe a un entorno familiar $\mathrm{y}$, los dispositivos más pequeños -el smartphone o la tableta- se destinan a un consumo individual (Teso \& Piñuel, 2014: 94).

Tal y como se afirma anteriormente, los más jóvenes tienden hacia nuevas formas de consumo hipertelevisión - fundamentadas en la multiplicidad de formatos y pantallas. El smartphone se ha erigido en el dispositivo predilecto de los adolescentes (Sádaba \& Vidales, 2015; Mascheroni \& Ólafsson, 2016), puesto que deja de ser un simple medio de comunicación para transformarse en un elemento "multiuso e interactivo" (Méndiz, De Aguilera \& Borges, 2011: 78). No en vano, lo utilizan no solo como herramienta comunicativa, sino también para otros múltiples usos: "empleo de redes sociales, juegos, aplicaciones y widgets" (Gaspar Herrero \& Cuesta Díaz, 2015: 523).

Queda patente que los adolescentes, primordialmente, utilizan las redes sociales. Su influencia entre los jóvenes es indiscutible (Livingstone, 2008; Nuñez, García \& Hermida, 2012; López de Atala \& Catalina, 2013). Entre las más empleadas, destaca YouTube. Esta plataforma digital de vídeos, relacionada originariamente con el mundo de los videojuegos, ha acumulado millones de visualizaciones de los youtubers que comparten videotutoriales o videoblogs sobre temáticas dispares (Chau, 2010; García, Catalina \& López de Ayala, 2016). Los youtubers, además, ejercen una gran influencia sobre los adolescentes (McRoberts, Bonsignore, Peyton \& Yarosh 2016); ya que estos últimos los toman como modelo.

En esencia, los adolescentes de la generación Z son "prosumidores" (Toffler, 1981: 262-263), porque se convierten en creadores de sus propios vídeos y los muestran públicamente en YouTube. En el campo de los videojuegos, por el contrario, las investigaciones realizadas determinan que existe un porcentaje menor de usuarios y su tipología es mayoritariamente masculina, con una clara tendencia hacia productos de naturaleza sexista y violenta (Anderson \& Bushman, 2001; Díez, 2009; Alcolea, 2014).

El consumo digital adolescente no se circunscribe solo al entretenimiento. El desarrollo de las relaciones sociales se configura como otro de sus principales rasgos. En ese sentido, resulta preciso subrayar el significativo crecimiento de redes sociales como Instagram y Facebook, utilizadas, fundamentalmente, con el fin de incrementar y afianzar sus relaciones personales (Colás, González \& De Pablos, 2013; Marcelino, 2015).

Esta gran influencia era ya destacada por las investigaciones pioneras en este ámbito (Livingstone, 2008; Livingstone, Haddon, Görzig \& Ólafsson, 2011; Boyd, 2014), y, además, se ha visto ratificada por los últimos datos del estudio del Observatorio Vasco de la Juventud (2016). Según dichas cifras, casi todos los jóvenes vascos de entre 15 y 29 años $-99 \%$ - son usuarios de alguna red social y se conectan diariamente.

$\mathrm{Si}$ bien el relieve que adquieren las redes sociales es común a ambos sexos, conviene reseñar la influencia que el género de los usuarios tiene en sus pautas de comportamiento. Las investigaciones más significativas realizadas en dicho marco (Espinar \& González, 2009; Estébanez \& Vázquez, 2013; Alonso, Rodríguez, Lameiras \& Carrera, 2015) evidencian que las chicas, además de utilizar más las redes sociales, interactúan con mayor frecuencia entre ellas e intercambian una cantidad de contenidos superior. Igualmente, su exposición pública suele ser mayor que la de los chicos. 
Las diferencias de género se reflejan, también, en las pautas de consumo mediático (Adriaens, Van-Damme \& Courtois, 2011; Alcolea, 2014) de chicos y chicas, respectivamente. Se trata, pues, de patrones de consumo opuestos y diferenciados entre sí. Así, mientras las chicas optan por series de ficción dramática, reality shows y programas del corazón; los chicos prefieren consumir contenidos relacionados con el deporte y el humor (Medrano \& Cortés, 2008; Masanet, 2016; Pibernat, 2017).

Los valores que transmiten los citados productos audiovisuales han sido objeto de estudio de numerosas investigaciones (Medrano, Aierbe Orejudo, 2009; Rodríguez Martínez, Medrano \& Martínez de Morentín, 2013; Korres \& Elexpuru, 2017) que se han centrado, sobre todo, en aspectos vinculados al género, la edad y el contexto cultural. Asimismo, se ha analizado profusamente la representación de género en series y filmes, productos culturales que ocupan un lugar relevante en el ocio de los adolescentes contemporáneos (Fedele \& García Muñoz, 2010; García Muñoz, Fedele, 2011; Álvarez, González de Garay \& Frutos, 2015).

Al hilo de lo expuesto, es incuestionable -tal y como afirma María Ángeles Gabino (2004)- que existe un vínculo notorio entre el consumo y la creación audiovisual adolescente y los espacios de ocio, por encima de los ámbitos formales y educativos. No obstante, la presencia de las Tecnologías de la Información y de la Comunicación (TIC) en el entorno escolar es cada vez mayor, lo que otorga al alumnado un fácil acceso a fuentes complementarias de información (Eynon \& Malmberg, 2011; Fernández-Planells \& Figueras-Maz, 2012; Solano, González \& López, 2013; Ciampa, 2014). En este contexto, Wikipedia se ha convertido en la principal herramienta de los escolares para obtener información en la Red (Salmerón, Cerdán \& Naumann, 2015; Tramullas, 2016; Valverde \& González, 2016).

\subsection{La transcendencia de la lengua en el entorno digital}

Pese a que la lengua es un factor determinante a la hora de analizar el uso y los hábitos de consumo y creación audiovisual adolescente -sobre todo, en sociedades bilingües como la guipuzcoana-, muy ocasionalmente adquiere la importancia que merece en las diversas investigaciones que examinan dicho objeto de estudio.
Hace ya dos décadas Jim Erickson (1998) alertaba de que la hegemonía de la lengua inglesa en Internet suponía una seria amenaza para las demás. En su artículo Cyberspeak: the Death of Diversity concluía que la Red, a pesar de ser una herramienta ideal para construir una comunidad internacional, podría estimular el posicionamiento del inglés como idioma global dominante.

Sin embargo, otros reputados lingüistas liderados por David Crystal (2005), opinan justamente lo contrario. Según esta influyente corriente, la posición del inglés está lejos de ser dominante, puesto que por efecto de la globalización la presencia de otros idiomas ha ido incrementándose progresivamente.

De todas formas, aunque Internet refleje cada vez con más nitidez la distribución de las lenguas en el mundo real y existan cientos de sitios importantes que reúnen todo tipo de información sobre las propias lenguas (Crystal, 2005: 118), lo cierto es que la amenaza que se cierne sobre las lenguas minoritarias es cada vez más palmaria. András Kornai (2013), por ejemplo, reviene del peligro de "muerte digital" que corren las lenguas no hegemónicas. Hoy en día, menos del 5\% de idiomas tiene acceso a la Red, lo que implica que una multitud de lenguas desaparecerá por efecto de la brecha digital.

El euskera cohabita con el castellano, lengua hegemónica, y con el inglés en el ámbito audiovisual. Si se tienen en consideración los últimos datos relativos al conocimiento de la lengua vasca, parece que hay motivos suficientes para ser optimista. Según la VI. Encuesta Sociolingüística de 2016, la cifra de vascoparlantes en la Comunidad Autónoma Vasca ha experimentado un notable incremento. Más de la mitad de la ciudadanía vasca conoce el idioma -el $33,9 \%$ de la población mayor de 16 años es vascoparlante y el $19,1 \%$ es vascoparlante pasivo- Asimismo, la hegemonía del modelo lingüístico D en Gipuzkoa -territorio en el que se centra la investigación- es irrebatible. Los datos de matriculación del curso 2017/2018 certifican lo expuesto. De los 28.817 alumnos matriculados en la ESO, 23.698 estudian en el modelo D. Nada más y nada menos, que el $82 \%$ del total. Por otro lado, 4.367 estudian en el modelo B, y tan solo 752 lo hacen en el modelo A.

No obstante, no hay correspondencia entre competencia lingüística y uso del euskera. Solamente el 30,6\% de los ciudadanos vascos usa el euskera en mayor o menor medida. Más de dos 
tercios de la población de la CAV - $69,4 \%$ - utiliza el castellano siempre o casi siempre. Asimismo, según el último informe de Soziolinguistika Klusterra (2017), el uso del euskera en el ámbito público en Euskal Herria ha sufrido un retroceso. En Gipuzkoa, concretamente, su uso ha descendido 1,5 puntos en el último lustro. Lo preocupante es que dicho descenso, además de en la capital donostiarra, se ha registrado en las poblaciones que tienen mayor conocimiento del idioma. Esta tendencia a la baja en el uso del euskera no es reciente, diversos estudios (Mujika, 2012; Iurrebaso, 2012) alertaban de la ralentización del crecimiento del uso del euskera al comienzo de la década.

En el contexto educativo, la situación es similar. En otras palabras, el uso del euskera entre el alumnado tampoco se corresponde con su conocimiento de la lengua vasca, es significativamente menor. Según se desprende del informe técnico Proyecto Arrue: Uso del euskera por el alumnado en el entorno escolar de la C.A.P.V. 2011-2015, el principal anhelo de los adolescentes es ser como las personas adultas. En consecuencia, los adolescentes adoptan la norma social de uso lingüístico de su entorno y se expresan principalmente en castellano (Gobierno Vasco, 2017: 130). El adolescente, por tanto, lejos del marco escolar, se inclina por el uso del idioma dominante en su entorno social. Y, esa circunstancia, influye sobremanera en la elección de la lengua en el consumo cultural e informativo que desarrolla.

\section{Metodología}

El diseño metodológico de este estudio combina un sondeo probabilístico cuantitativo y dinámicas de grupo que aportan información cualitativa.

La investigación recoge los datos de 2.426 encuestas realizadas a adolescentes (de 12 a 16 años) que cursan sus estudios de Educación Secundaria Obligatoria (de $1^{\circ}$ a $4^{\circ}$ de la ESO) en los modelos lingüísticos $\mathrm{B}(50 \%$ en castellano y $50 \%$ en euskera) y D (íntegramente en euskera) del Territorio Histórico de Gipuzkoa.

Con el fin de alcanzar los objetivos del estudio se diseñó un cuestionario autorellenable en euskera con cien preguntas divididas en seis grandes bloques: el primero de ellos se ocupa del tiempo que les dedican los adolescentes a sus actividades de ocio y al consumo audiovisual. El segundo, rastrea el consumo de contenidos audiovisuales tradicionales (ficción, programas de televisión, deporte, actualidad, música, videoclips y videojuegos). El tercero, aborda los nuevos formatos de Internet (youtubers, videotutoriales y comunidades virtuales). El cuarto, analiza el uso de las redes sociales, el quinto, la creación y difusión de contenidos, y el sexto, el consumo de fuentes no oficiales para completar los conocimientos adquiridos en la formación reglada. La taxonomía de las preguntas del cuestionario es heterogénea, 57 de ellas plantean respuestas múltiples, 28 son de respuesta única y las otras 15 son de respuesta abierta.

La encuesta contempla ocho variables de cruce (potencialmente discriminantes de comportamiento): el género, el curso, la titularidad del centro, el estatus social de las familias, el control social y tecnológico de los adolescentes, el acceso a la tecnología, la distribución comarcal y el uso del euskera.

El universo de estudio son 28.817 chicos y chicas distribuidos en 108 centros de ESO de modelo B y D y la unidad ha sido el grupo. Se optó por un muestreo por conglomerados, estratificado por afijación proporcional teniendo en cuenta la distribución geográfica de los centros y los niveles educativos. La muestra es una selección aleatoria de 60 centros, un total de 120 grupos, 30 por cada curso. El error muestral máximo es del $+/-1,90 \%$ para el conjunto del territorio de Gipuzkoa y no supera el $+/-7 \%$ en ninguna de las comarcas. El nivel de confianza estadístico es del 95\% (en el supuesto más desfavorable de $\mathrm{p}=\mathrm{q}=0,5$ ).

El trabajo de campo se realizó entre diciembre de 2016 y enero de 2017. Las encuestas se realizaron en el aula bajo la dirección de uno de los investigadores y con la presencia del profesor o tutor de cada grupo.

El estudio cualitativo se basa en cuatro grupos de discusión o focus group, uno por cada curso de la ESO, formados por 8 alumnos, 4 chicos y 4 chicas, y que se realizaron en las comarcas de Donostialdea, Oarso-Behe Bidasoa, Goierri y Debagoiena. El objetivo era recoger información de tipo actitudinal, comportamental y proyectiva de cada colectivo en base a un guion que recoge los temas clave del sondeo cuantitativo.

\section{Resultados y conclusiones}

No resulta tarea fácil presentar de manera concisa los resultados de la investigación si se tiene en cuenta el tamaño de la muestra, la cantidad de 
variables analizadas y los abundantes datos obtenidos. Por consiguiente, en lugar de optar por un apartado clásico y extenso que muestre de manera descriptiva el conjunto de los resultados, y posteriormente, dar paso a las conclusiones finales, se ha decidido integrar de manera gradual en las conclusiones los resultados más significativos de la investigación.

\subsection{El smartphone, la herramienta indispensable}

En primer término, resulta imprescindible subrayar que el smartphone es la herramienta esencial de los adolescentes guipuzcoanos. Es, junto con la televisión, el medio de consumo cultural preferente, muy por encima del ordenador y la tableta. Si bien es cierto que la televisión concentra todavía el consumo audiovisual estándar (series, películas, realities, deporte...), el teléfono inteligente es ya la opción predilecta de los más jóvenes para el consumo de videoclips, videojuegos, youtubers, etc.

Asimismo, los adolescentes son prosumidores. Además de consumir productos culturales, crean los suyos propios. Y, para ello, utilizan casi exclusivamente el smartphone. Esas piezas audiovisuales las comparten mediante Instagram y Youtube, principalmente. Sin embargo, resulta preciso hacer una distinción en este punto. Cuando las productos audiovisuales tienen carácter público, no hay apenas diferencia porcentual en el uso de Youtube $(45,2 \%)$ e Instagram (45\%). Pero, cuando esas piezas se comparten de manera privada, Instagram $(81,8 \%)$ es la opción elegida mayoritariamente.

\subsection{El castellano, lengua de consumo en un territorio fracturado lingüísticamente}

Aunque los adolescentes guipuzcoanos objeto de esta investigación están inmersos en un entorno bilingüe y son capaces de comunicarse en euskera y castellano $-\mathrm{y}$ muchos de ellos, también en inglés-, su consumo cultural e informativo se desarrolla eminentemente en castellano. Es innegable que existen múltiples condicionamientos que inciden en ello: la preeminencia de las lenguas hegemónicas en la Red, la escasa presencia del euskera en las salas cinematográficas, la sugestiva oferta de las televisiones de pago... Pese a todo, la disonancia entre competencia lingüística $\mathrm{y}$ consumo es más que significativa.
$\mathrm{Si}$ nos atenemos a los resultados de la investigación, el $70,17 \%$ de los contenidos que consumen los adolescentes guipuzcoanos suelen ser en castellano. Los contenidos consumidos en euskera, por el contrario, suponen solamente el $19,96 \%$ del total. La presencia del inglés, por su parte, resulta significativa; puesto que los jóvenes guipuzcoanos consumen uno de cada diez contenidos $(9,87 \%)$ en dicha lengua. Las cifras son muy elocuentes y evidencian de manera diáfana que el castellano es la lengua de consumo preferida de los adolescentes guipuzcoanos.

Sin embargo, la hegemonía del castellano como lengua de consumo no es, en absoluto, uniforme en todas las zonas del territorio. Existe, de hecho, una enorme brecha entre las siete comarcas guipuzcoanas. Los adolescentes de las comarcas de Oarso-Behe Bidasoa y Donostialdea, en concreto, consumen mucho más en castellano y mucho menos en euskera. La situación es muy diferente en Tolosaldea, Urola Kosta, Debabarrena, Debagoiena y Goierri. Si bien en estas comarcas el consumo de contenidos audiovisuales en castellano sigue siendo mayor, es proporcionalmente inferior al de las citadas con anterioridad. Asimismo, los porcentajes de consumo de contenidos audiovisuales en euskera superan la media y son más altos que los de Oarso-Behe Bidasoa y Donostialdea.

El caso de la exitosa serie en euskera Go!azen refleja perfectamente esta fractura comarcal. Si tenemos en cuenta las preferencias de los adolescentes guipuzcoanos, Go!azen sería su cuarta serie de humor favorita, dado que el $42,4 \%$ de los jóvenes admite que la ve regularmente. De todos modos, tal y como se ha señalado, los porcentajes varían mucho de una comarca a otra. Así, mientras en Tolosaldea $(53,8 \%)$, Urola Kosta $(51,4 \%)$, Debabarrena $(\% 56,4), \quad$ Debagoiena $(\% 52,3 \%)$ y Goierri $(51,5 \%)$ el porcentaje de adolescentes que consume Go!azen supera ampliamente la media, en Donostialdea $(36,1 \%)$ y Oarso Behe.Bidasoa $(\% 22,6)$ está muy por debajo.

La afectividad respecto al euskera constituye un factor clave a la hora de interpretar esta fractura comarcal. No en vano, los adolescentes que habitualmente usan el euskera en sus relaciones sociales y lo emplean también tanto en el ámbito tecnológico como en el académico - los jóvenes de las comarcas en las que más se habla el euskera, en definitiva- consumen más contenidos audiovisuales en euskera, y viceversa. Por otra parte, los adolescentes de Oarso-Behe Bidasoa y 
Donostialdea, los jóvenes que menos contenidos audiovisuales consumen en euskera, son los que más consumen en inglés.

\subsection{Diferencias de género tanto en el consumo como en la creación de contenidos}

Los chicos y las chicas interaccionan de manera claramente diferenciada con los contenidos audiovisuales en general, y por extensión, con aquellos que son en euskera. Esta diversidad marcada por el género se manifiesta en diferentes apartados:

- Uso de los medios. Las chicas utilizan más el ordenador, la tableta y, sobre todo, el smartphone. Dado que usan con más frecuencia el teléfono inteligente, interaccionan on line (Twitter, Facebook, Snapchat, WhatsApp...) considerablemente más que los chicos. Además, acuden con más asiduidad a las salas cinematográficas, consumen más televisión y utilizan más la plataforma Youtube. Los chicos, en cambio, escuchan más la radio -programas de deportes, especialmente- $\mathrm{y}$ juegan más con las videoconsolas.

- Consumo de contenidos audiovisuales. A la hora de visionar películas -tanto en el cine como en la televisión- las chicas se inclinan por el género romántico y los melodramas, en tanto que los chicos optan más por el género de acción y la fantasía. Asimismo, las chicas consumen también más programas informativos -excepto los de temática deportiva que tienen un público juvenil eminentemente masculino-, más realities, videoclips y programas del corazón, mientras que los chicos destacan, sobre todo, por su consumo de contenidos deportivos. En cuanto al seguimiento de youtubers y el consumo de videotutoriales, las chicas se inclinan mayoritariamente por aquellos que se centran en contenidos vinculados al mundo de la belleza y la moda, la gastronomía y los viajes. Los chicos, por su parte, tienden hacia aquellos relacionados con los videojuegos.

- $\quad$ Si nos referimos al consumo de contenidos audiovisuales en euskera, cabe reseñar que las chicas consumen más productos culturales en dicha lengua que los chicos. En el plano de la televisión, por ejemplo, solo el $29,1 \%$ de las chicas manifiesta que no ve ETB1, la cadena íntegramente en euskera. En el caso de los chicos, el porcentaje que no ve ETB1 se eleva al $45,8 \%$. En otras palabras, casi la mitad de los chicos no consume los programas en euskera de ETB1. La audiencia del programa Go!azen evidencia de manera nítida esta realidad, puesto que la audiencia femenina $(59,3 \%)$ duplica la masculina $(26,7 \%)$.

- Conocimiento para la creación de contenidos audiovisuales. Consecuencia lógica de su mayor uso interactivo del smartphone, las chicas poseen más conocimientos en torno a las herramientas destinadas a la creación audiovisual registro, edición y emisión vía on line- $\mathrm{y}$ son, también, más hábiles en el uso de los medios.

- Producción audiovisual. Las chicas crean más contenidos audiovisuales - fotografías y vídeos, sobre todo- y los suben con mayor frecuencia a la Red. Los chicos destacan, en gran medida, en la creación de tutoriales sobre videojuegos. Es preciso subrayar que la inmensa mayoría de estos contenidos audiovisuales tienen como idioma el castellano. La creación audiovisual en euskera del target es excepcional, realmente exigua.

\subsection{Consumo y creación en función de la edad}

El incremento de edad conlleva, como es lógico, cambios en el uso de los medios, en los gustos y consumo de contenidos, en el conocimiento de los medios de creación y en la producción de contenidos audiovisuales. En términos generales, a medida que el adolescente cumple años $-\mathrm{y}$ va superando los cursos sucesivos de la ESO, aumentan de forma prácticamente exponencial casi todos estos índices, de forma manifiesta el uso y conocimiento del manejo del smartphone, con todas sus potencialidades, en especial las vinculadas a la creación audiovisual.

En el terreno del consumo, a medida que los adolescentes se acercan a la edad adulta, tienden a ver más películas y series. Igualmente, consumen más realities e información. Sin embargo, acuden menos a las salas cinematográficas. Los cambios, empero, no se materializan solamente en el 
consumo de contenidos, sino también, en la forma de consumirlos. La costumbre de ver la televisión en familia desciende significativamente y se impone la tendencia por un ocio más individualizado. De este modo, los jóvenes de los últimos cursos optan por consumir las películas y las series on line solos o con amigos. Precisamente, en este consumo de filmes y series on line, la diferencia entre el alumnado de primero $(24,1 \%)$ y de cuarto $(39,6 \%)$ de ESO es de, nada más y menos, un $15,5 \%$.

En esta tesitura, tal y como se ha mencionado con anterioridad, el empleo del smartphone se acentúa. Y, asimismo, los jóvenes dedican mucho más tiempo a chatear, twittear $\mathrm{y}$, sobre todo, a enviarse mensajes y contenidos a través de WhatsApp. Así, mientras los alumnos de primero de ESO invierten los fines de semana una hora en dichos menesteres, los de cuarto de ESO les dedican más de media hora (97 minutos).

Por otra parte, en lo referente a la creación, a medida que pasan los años, los jóvenes acumulan más conocimiento para producir contenidos audiovisuales. Si en primero de ESO casi una tercera parte de ellos $(30,8 \%)$ no tiene conocimiento suficiente para crear contenidos audiovisuales, en cuarto de ESO esa cifra baja casi a la mitad $(18,1 \%)$.

La edad tiene también cierta repercusión en el consumo de contenidos audiovisuales en euskera, puesto que el alumnado de los últimos cursos de la ESO consume menos. Mientras el $20,8 \%$ del alumnado de primero consume entre un $25 \%$ y un $50 \%$ contenidos audiovisuales en euskera, solo el $16,7 \%$ del alumnado de tercero lo hace en esa franja.

\subsection{Más que creadores, consumidores}

La generación $\mathrm{Z}$ es, por definición, prosumidora. No solo consume contenidos audiovisuales, sino que también los produce. Los adolescentes guipuzcoanos son de la misma naturaleza. Según los datos que se desprenden de la investigación, el $71,7 \%$ de los jóvenes guipuzcoanos tiene aptitud para crear contenidos. El 31,5\% del alumnado, además, es capaz de utilizar una cámara, editar un vídeo en el ordenador y contar una historia a través de imágenes. En suma, es competente para crear contenidos audiovisuales de cierta complejidad. No obstante, a pesar de que la mayoría de los adolescentes guipuzcoanos tiene conocimientos suficientes -sencillos o más especializados- para crear y compartir contenidos, solamente unos pocos son creadores. De hecho, más de la mitad del alumnado $(57,2 \%)$ nunca ha subido un vídeo a Internet.

Esta tendencia se repite también con los youtubers. Pese a que el $61,4 \%$ de los jóvenes guipuzcoanos admite ser seguidor de algún youtuber, solo el $7 \%$ de los encuestados reconoce ejercer o haber ejercido alguna vez como youtuber. Ocurre algo similar con los videotutoriales. El $62,4 \%$ del alumnado consume videotutoriales, pero únicamente el $12,3 \%$ ha creado alguno. Teniendo en cuenta todos estos datos, parece que entre los adolescentes guipuzcoanos no existe demasiado interés por la creación. Es, por cierto, la actividad de ocio a la que le dedican menos tiempo. Sin duda, los adolescentes guipuzcoanos son más consumidores que creadores.

Si la creación de contenidos audiovisuales resulta escasa en general, la situación se agrava aún más en el caso de que estos sean en euskera. Muestra de ello es que, entre los adolescentes guipuzcoanos que crean contenidos, el $57 \%$ no produce ninguno en euskera. En consecuencia, la mayor parte de los contenidos audiovisuales creados son íntegramente en castellano. La inmensa mayoría de los adolescentes guipuzcoanos que ejercen de youtubers lo hacen también en castellano $(78,2 \%)$. Solo un $4,5 \%$ de ellos utiliza exclusivamente el euskera. Con los videotutoriales sucede algo similar. Mientras que el $60,7 \%$ de los adolescentes crea videotutoriales en castellano, solamente el 10,4\% los produce en euskera. Según la información que aportan los focus group realizados con los propios alumnos, uno de los motivos principales del exiguo espacio que ocupa el euskera en las creaciones audiovisuales se debe a que los jóvenes crean sus contenidos teniendo en cuenta los amplios públicos potenciales que les brindan las lenguas hegemónicas.

\subsection{Bajo consumo de los medios audiovisuales tradicionales en euskera}

Los adolescentes guipuzcoanos, en gran medida, realizan un consumo más bien discreto de los medios audiovisuales tradicionales en euskera. $\mathrm{Si}$ se tiene en cuenta que los jóvenes encuestados estudian en los modelos $\mathrm{B}$ y $\mathrm{D}$, resulta especialmente significativo que uno de cada tres $(37,4 \%)$ no vea ETB1, la cadena pública de referencia en euskera. No obstante, los resultados de Hamaika Telebista son aún más desalentadores, 
puesto que el $96,3 \%$ del alumnado afirma que no consume sus programas.

Las televisiones locales que emiten en euskera tienen una aceptación similar, más baja aún si cabe. Goiena Telebista es, entre estas últimas, la que muestra mejores índices de audiencia. Pese a todo, dos de cada tres adolescentes $(67,8 \%)$ no la ven. Cabe reseñar, sin embargo, que tiene en su parrilla programas dirigidos a adolescentes de relativo éxito. Un claro exponente de ello es Kantari, un programa de karaoke con canciones en euskera que aglutina al $15,9 \%$ del alumnado adolescente de la comarca. Las audiencias de las televisiones locales como Goierri Telebista, 28. Kanala y Erlo son todavía más pequeñas.

Aunque ver la televisión -paulatinamente se va imponiendo la Smart TV en castellano- aún sigue siendo una de las actividades principales de los nativos digitales, la televisión en euskera ha perdido audiencia. La competencia de las empresas de entretenimiento que ofrecen contenido multimedia bajo demanda en lenguas hegemónicas y de las cadenas de televisión generalistas constituye uno de los múltiples factores que explican su caída. Incentivar la producción propia de programas de calidad en euskera destinados a los jóvenes podría ser una de las soluciones para evitar que la perdida sea irreversible.

\section{Conclusiones}

Las actividades prioritarias de ocio de los adolescentes guipuzcoanos están relacionadas con las nuevas tecnologías. Asimismo, la estimulante oferta de contenidos audiovisuales de las lenguas hegemónicas -sobre todo, del castellano- en la Red condicionan en extremo el consumo digital en euskera de los jóvenes guipuzcoanos.

Ante esta inédita situación, existen demasiadas debilidades. La oferta de contenidos audiovisuales en euskera es muy escasa. Y, dicha oferta, en la mayoría de los casos, resulta totalmente desconocida para los más jóvenes. De hecho, no hay un canal lo suficientemente popular para publicitarla. De la parte cualitativa de esta investigación se desprende, además, que la exigua oferta de contenidos audiovisuales en euskera es poco atractiva para los adolescentes. Los jóvenes guipuzcoanos que muestran poca empatía hacia el euskera tienen una actitud negativa respecto a los contenidos audiovisuales en esa lengua; una actitud que va desde el desinterés al rechazo expreso. Los adolescentes más empáticos no garantizan el consumo de los contenidos audiovisuales en euskera per se, y productos de ocio de calidad. A su vez, el target manifiesta más interés por el consumo que por la creación.

La fractura comarcal respecto al consumo y creación de contenidos audiovisuales en euskera, además, dificulta sobremanera una política común sobre la materia. Y, asimismo, antes de implementar cualquier plan que aborde la cuestión, conviene tener en cuenta también los diferentes patrones de género que presentan los adolescentes guipuzcoanos en su interacción con los productos audiovisuales.

En definitiva, existe una demanda formada de nativos digitales vascoparlantes que tienen acceso a los medios para el consumo y la creación audiovisual, con alta actividad en las redes sociales, que no ven satisfechas sus inquietudes por una oferta insuficiente y relativamente desconocida. El principal reto consiste en atraerlos y fomentar en ellos tanto el consumo como la creación de los contenidos audiovisuales en euskera.

Pese a que el fin es evidente, la tarea se antoja harto complicada. No obstante, la puesta en marcha de políticas públicas eficientes y perdurables que promuevan el uso del euskera entre los adolescentes guipuzcoanos a la hora de consumir, crear y compartir contenidos audiovisuales en los nuevos medios resulta indefectible y urgente. Igualmente, estas medidas deberían complementarse con otras que fomenten el consumo de productos audiovisuales en euskera destinados a los jóvenes en los medios tradicionales.

A la luz de las conclusiones que se extraen de esta investigación, el smartphone debe ser la piedra angular. Es, indudablemente, la herramienta que puede mitigar los peligros detectados en el estudio de campo, así como el medio idóneo para sacar provecho de las oportunidades que ofrece este target. En este sentido, estimular el consumo en euskera es imprescindible. El sector audiovisual debe efectuar un especial esfuerzo para trabajar de manera colectiva y ofrecer contenidos de calidad. Y, para ello, el apoyo de las instituciones se torna esencial. Resulta primordial desarrollar nuevas estrategias con la finalidad de que tales contenidos de calidad sean de conocimiento público, accesibles y se distribuyan adecuadamente. A su vez, es necesario impulsar e incentivar la creación en euskera entre los jóvenes, dado que otro de los 
principales retos futuros consiste en llevar al terreno práctico la competencia tecnológica.

Llegados a este punto, cualquier tentativa de fomentar la producción audiovisual en euskera debería tener en cuenta ciertos aspectos. En primer lugar, se han de encontrar fórmulas de estímulo creativo y de consumo que impliquen a los jóvenes de forma espontánea y voluntaria, lejos del ámbito educativo. Y, además, estas deberían estar ligadas a las actividades de ocio y entrar en sinergia con su participación habitual en las redes sociales.

Asimismo, conviene prestar la atención necesaria a la fractura comarcal en torno al consumo y la creación de contenidos audiovisuales en euskera en el territorio. Existe una brecha comarcal entre los adolescentes de Donostialdea y Oarso Behe-Bidasoa y los del resto de Gipuzkoa. No es una cuestión de fácil arreglo, y resulta patente que el contexto sociolingüístico tiene una influencia notoria. Así pues, antes de implementar las políticas públicas mencionadas con anterioridad, sería oportuno adecuarlas a las diversas realidades sociolingüísticas que conviven en el territorio con el fin de que sean efectivas.

Las instituciones, la Diputación Foral de Gipuzkoa especialmente, han puesto ya en marcha iniciativas encaminadas a fomentar entre los adolescentes el consumo y la creación de contenidos audiovisuales en euskera. Se ha creado un laboratorio de audiovisuales en euskera, se han estrechado los vínculos con el sector audiovisual del territorio y se han articulado diversos proyectos transmedia. Resulta fundamental ahondar y profundizar en los pasos dados y urge tomar otras medidas como crear una plataforma digital común que aúne el conjunto de la producción audiovisual en euskera, la visibilice y facilite tanto el consumo como la creación de los jóvenes guipuzcoanos. En este nuevo contexto en el que están en riesgo tanto la pervivencia de los productos audiovisuales en euskera como la propia lengua, resulta esencial la interacción y colaboración entre las empresas del sector audiovisual en euskera y las instituciones.

\section{Referencias}

Adriaens, F., Van-Damme, E., \& Courtois, C. (2011). The spatial and social contexts of television-viewing adolescents. Poetics, 39 (3), 205-227. https://doi.org/10.1016/j.poetic.2011.03.001.

Alcolea, G. (2014). Análisis del consumo adolescente, con variables de género, de series y videojuegos: Formas de acceso y actividad multitarea. VI Congreso Internacional Latina de Comunicación Social, Universidad de la Laguna, 1-14. Recuperado de: https://bit.ly/2wAs3j8

Alonso, P., Rodríguez, Y., Lameiras, M., \& Carrera, M.V. (2015). Hábitos de uso en las redes sociales de los y las adolescentes: Análisis de género. Revista de Estudios e Investigación en Psicología y Educación, 13, 54-57. https://doi.org/10.17979/reipe.2015.0.13.317

Álvarez, C., González-de-Garay, B., \& Frutos, F.J. (2015). Representación de género. Las películas españolas contemporáneas de adolescentes (2009-2014). Revista Latina de Comunicación Social, 70, 934-960. https://doi.org/10.4185/RLCS-2015-1079

Anderson, C.A., \& Bushman, B.J. (2001). Effects of violent video games on aggressive behavior, aggressive cognition, aggressive affect, physiological arousal, and prosocial behavior: A meta-analytic review of the scientific literature. Psychological Science, 12 (5), 353-359. https://doi.org/10.1111/1467-9280.00366

Aranda, D., Sánchez, J., Tabernero, C., \& Tubella, I. (2010). Los jóvenes del siglo XXI: Prácticas comunicativas y consumo cultural. II Congreso Internacional AE-IC Comunicación y desarrollo en la era digital, Universidad de Málaga (1-20). https://bit.ly/2rzUHMk

Boyd, D. (2014). It's complicated: The social lives of networked teens. New Haven (Connecticut): Yale University Press.

Buckingham, D., \& Martínez, J.B. (2013). Interactive youth: New citizenship between social networks and school settings. [Jóvenes interactivos: Nueva ciudadanía entre redes sociales y escenarios escolares]. [Comunicar, 20 (40), 10-14. https://doi.org/10.3916/C40-2013-02-00

Castells, M. (2006). La sociedad red: una visión global. Madrid: Alianza.

Catalina, B., López-de-Ayala, M.C., \& García, A. (2014): Los riesgos de los adolescentes en Internet: los menores como actores y víctimas de los peligros de Internet. Revista Latina de Comunicación Social, 69, 462-485. https://doi.org/10.4185/RLCS-2014-1020 
Colás, P., González, T., \& De-Pablos, J. (2013). Juventud y redes sociales: Motivaciones y usos preferentes. Comunicar, 20 (40), 15-23. https://doi.org/10.3916/C40-2013-02-01

Chau, C. (2010). YouTube as a participatory culture. New Directions for Youth Development, 128, 65-74. https://doi.org/10.1002/yd.376

Ciampa, K. (2014). Learning in a mobile age: An investigation of student motivation. Journal of Computer Assisted Learning, XXX (1), 82-96. https://doi.org/10.1111/jcal.12036

Crystal, D. (2005). La revolución del lenguaje. Madrid: Alianza.

Díez, E. (2009). Sexismo y violencia: La socialización a través de los videojuegos. Feminismo/s, 14, 35-52. https://bit.ly/2Iu4yx1

Durán, M. \& Martínez-Pecino, R. (2015). Ciberacoso mediante teléfono móvil e Internet en las relaciones de noviazgo entre jóvenes. Comunicar 22 (44), 159-167.https://doi.org/10.3916/C44-2015-17Principio del formularioFinal del formulario

Espinar, E., \& González, M.J. (2009). Jóvenes en las redes sociales virtuales: Un análisis exploratorio de las diferencias de géneros. Feminismo/s, 14, 87-106. Recuperado de: https://bit.ly/2KTBmxJ

Estébanez, I., \& Vázquez, N. (2013). La desigualdad de género y el sexismo en las redes sociales. Vitoria-Gasteiz: ONA. Recuperado de: https://bit.ly/2rCjCOD

Erickson, J. (1998). Cyberspeak: the death of diversity. Asiaweek, 15.

Eynon, R., \& Malmberg, L. (2011). A typology of young people's Internet use: Implications for education. Computers \& Education, 56 (3), 585-595. https://doi.org/10.1016/j.compedu.2010.09.020

Fedele, M., \& García-Muñoz, N. (2010). El consumo adolescente de la ficción seriada. Vivat Academia, 111, pp. 4764. https://doi.org/10.15178/va.2010.111.47-64

Fernández-Cruz F.J., \& Fernández-Díaz, M.J. (2016). Los docentes de la Generación Z y sus competencias digitales. Comunicar 24 (46), 97-105. https://doi.org/10.3916/C46-2016-10

Fernández-Planells, A., \& Figueras, M. (2012). Internet en las tareas escolares, ¿obstáculo u oportunidad? El impacto de la Red en los hábitos de estudio de alumnos de Secundaria de Barcelona y Lima. Sphera Pública, (12), 161-182. Recuperado de: https://bit.ly/2ELbkIy

- (2014). De la guerra de pantallas a la sinergia entre pantallas: El multitasking en jóvenes. In A. Huertas, \& M.

Figueras (Eds.), Audiencias juveniles y cultura digital (pp.87-107). Bellaterra (Barcelona): Institut de la Comunicació. Universitat Autònoma de Barcelona. Recuperado de: https://bit.ly/2Kfa7N2

Gabelas, J.A. \& Lazo, C.M. (2008). Modos de intervención de los padres en el conflicto que supone el consumo de pantallas. Revista Latina de Comunicación Social, 63, 238-252. https://doi.org/10.4185/RLCS-632008-763-238-25

Gabino, M.A. (2004). Children and young people as virtual and interactive users and receivers. [Niños y jóvenes como usuarios-receptores virtuales e interactivos]. Comunicar, 22, 120-125. Recuperado de: https://bit.ly/2KcOXyT

García, A., Catalina, B., \& López-de-Ayala, M.C. (2016). Adolescents and YouTube: Creation, participation and consumption. Prisma Social, 1, 60-89. https://bit.ly/2I8BLhV

García-Muñoz, N., \& Fedele, M. (2011). The Teen Series and the Young Target. Gender Stereotypes In Television Fiction Targeted to Teenagers. Observatorio (OBS*), 5(1), 215-226. https://doi.org/10.15847/obsOBS512011389

Gaspar Herrero, S. \& Cuesta Díaz, V. (2015). Adicción al smartphone. Análisis motivacional de uso entre nativos digitales. Opción 31 (4), 517-531.

Gewerc, A., Fraga, F., \& Rodes, V. (2017). Niños y adolescentes frente a la competencia digital. Entre el teléfono móvil, youtubers y videojuegos. Revista Interuniversitaria de Formación del Profesorado, 89, 171-186. Recuperado de: https://bit.ly/2rBmpZf

Howe, N., \& Strauss, W. (2000). Millenials Rising. The next great generation. New York: Vintage Books. Gobierno Vasco \& Soziolinguistika Klusterra. (2017). Proyecto Arrue: uso del euskera por el alumnado en el entorno escolar de la C.A.P.V. 2011-2015. Recuperado de: https://bit.ly/2J4bylg

Iurrebaso, I. (2012). Euskararen erabilera zertan den. Premiazko diagnostiko bat datuen argitan [The use of Basque language. An urgent diagnosis in the light of the data]. BAT Soziolinguistika 84 (3), 103-120. Recuperado de: https://bit.ly/2HeyMPP

Kornai, A. (2013). Digital Language Death. PloS ONE 8 (10), 1-11. Recuperado de: https://bit.ly/2121pYR 
Korres, O., \& Elexpuru, I. (2017): Análisis de los valores percibidos por los adolescentes en el medio televisivo. Valores percibidos por adolescentes en la TV. Infancia y Aprendizaje: Journal for the Study of Education and Development, 40 (4), 782-811. https://doi.org/10.1080/02103702.2017.1370821

Livingstone, S. (2008). Taking risky opportunities in youthful content creation: Teenagers' use of social networking sites for intimacy, privacy and self-expression. New Media \& Society, 10 (3), 393-411. https://doi.org/10.1177/1461444808089415

Livingstone, S., Haddon, L., Görzig, A., \& Ólafsson, K. (2011). Risks and safety on the Internet: The perspective of European children: Full findings and policy implications from the EU Kids Online survey of 9-16 year olds and their parents in 25 countries. LSE, London: EU Kids Online. Recuperado de: https://bit.ly/1jOA4AL

Livingstone, S., \& Helsper, E. (2010): Balancing Opportunities and Risks in Teenagers. Use of the Internet: the Role of Online Skills and Internet self-efficacy. New Media \& Society, 12 (2), 309-329. https://doi.org/10.1177/1461444809342697

García, A., López-de-Ayala, M., \& Catalina, B. (2013). Hábitos de uso en internet y en las redes sociales de los adolescentes españoles. Comunicar 21 (41), 195-204. https://doi.org/10.3916/C41-2013-19

Marcelino, G.V. (2015). Migración de los jóvenes españoles en redes sociales, de Tuenti a Facebook y de Facebook a Instagram. La segunda migración. Icono 14, volumen 13 (2), 48-72. Doi: https://doi.org/10.7195/ri14.v13i2.821.

McRoberts, S., Bonsignore, E., Peyton, T., \& Yarosh, S. (2016). Do It for the viewers!: Audience engagement behaviors of young YouTubers. In Proceedings of the The 15th IDC International Conference on Interaction Design and Children, 334-343. https://doi.org/10.1145/2930674.2930676

Marín, I., Yaguana, H.A., \& Barrazueta, P. (2013): Estudio sobre el uso y hábitos de consumo de medios de comunicación de la adolescencia en Loja (Ecuador). V Congreso Internacional Latina de Comunicación Social, Universidad de la Laguna, 1-13. Recuperado de: https://bit.ly/2twShiv

Masanet, M.J. (2016). Pervivencia de los estereotipos de género en los hábitos de consumo mediático de los adolescentes: Drama para las chicas y humor para los chicos. Cuadernos.info, 39, 39-53. https://doi.org/10.7764/cdi.39.1027

Mascheroni, G., \& Ólafsson, K. (2016). The mobile Internet: Access, use, opportunities and divides among

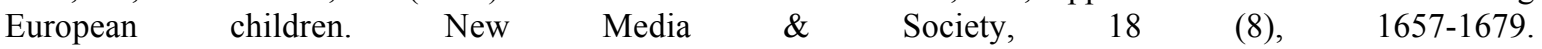
https://doi.org/10.1177/1461444814567986

Medrano, C., \& Cortés, A. (2008): ¿Persisten los estereotipos sociales en la dieta televisiva de los adolescentes? Comunicar, 16 (31), 381-386. https://doi.org/10.3916/c31-2008-03-022

Medrano, C., Aierbe, A., \& Orejudo, S. (2009). El perfil de consumo televisivo en adolescentes: Diferencias en función del sexo y estereotipos sociales. Infancia y Aprendizaje, 32 (3), 293-306. https://doi.org/10.1174/021037009788964150

Méndiz, A., De-Aguilera, M., \& Borges, E. (2011). Young people's attitudes towards and evaluations of mobile. [Actitudes y valoraciones de los jóvenes ante la TV móvil]. Comunicar, 36, 77-85. https://doi.org/10.3916/C36-2011-02-08

Montes, M., García, A., \& Menor, J. (2018). Teen videos on YouTube: Features and digital vulnerabilities. [Los vídeos de los adolescentes en YouTube: Características y vulnerabilidades digitales]. Comunicar, 26 (LIV), 61-69. https://doi.org/10.3916/C54-2018-06

Mujika, M. (2012). Euskararen ezagutza eta erabilera, motibazio eta borondate kontua [The knowledge and use of Basque language, a matter of motivation and willingness]. BAT Soziolinguistika 84 (3), 175-182. Recuperado de: https://bit.ly/2J6itdN

Núñez, P., García, M.L., \& Hermida, L.A. (2012): Tendencias de las relaciones sociales e interpersonales de los nativos digitales y jóvenes en la web 2.0. Revista Latina de Comunicación Social, 67, 179-204. https://doi.org/10.4185/RLCS-067-952-179-206

Observatorio Vasco de la Juventud (2016). La juventud vasca en las redes sociales. Vitoria: Gobierno Vasco, Departamento de Educación, Política Lingüística y Cultura. Recuperado de: https://bit.ly/2I7G1hY

Pibernat, M. (2017). ¿Nuevas socializaciones, viejas cuestiones? Adolescencia y género en la era audiovisual. Investigaciones feministas: papeles de estudios de mujeres, feministas y de género, 8 (2), 529-544. https://doi.org/10.5209/INFE.54976

Rodríguez-Martínez, A., Medrano, C., Aierbe, A., \& Martínez-de-Morentin, J.I. (2013): Television Viewing Habits and the Values Perceived by Adolescents: A Cross-cultural Study. Revista de Educación, 361, 513-538. 
Sádaba, C., \& Vidales, M.J. (2015). El impacto de la comunicación mediada por la tecnología en el capital social: adolescentes y teléfonos móviles. Virtualis, 11(1), 75-92. Recuperado de: https://bit.ly/2KRsoku

Salmerón, L., Cerdán, R., \& Naumann, J. (2015). ¿Cómo navegan los adolescentes en Wikipedia para contestar preguntas? Infancia y Aprendizaje, 38 (2), 435-471. https://doi.org/10.1080/02103702.2015.1016750

Solano, I. M., González, V., \& López, P. (2013). Adolescentes y comunicación: las TIC como recurso para la interacción social en educación secundaria. Pixel-Bit, 42, 23-35. Recuperado de: https://bit.ly/2KVdeLa

Soziolinguistika Klusterra. (2017). Hizkuntzen erabileraren kale-neurketa. Euskal Herria, 2016. Recuperado de: https://bit.ly/2lyP6Cx

Teso, G., \& Piñuel, J.L. (2015): Multitarea, Multipantalla y Práctica social del consumo de Medios entre los jóvenes de 16 a 29 años en España”. En Arrojo, M.J., \& Piñuel, J.L. (Coords.) Contenidos digitales y multipantalla, (pp. 93-108). Tenerife: Sociedad Latina de Comunicación. Recuperado de: https://bit.ly/2yHzHtd Toffler, A (1981). La tercera ola. Barcelona, Plaza \& Janes.

Tramullas, J. (2016). Competencias informacionales básicas y uso de Wikipedia en entornos educativos. Gestión de la Innovación en Educación Superior, 1(1), 79-95. Recuperado de: https://bit.ly/2jQabru

Valverde, D., \& González, J. (2016). Búsqueda y selección de información en recursos digitales: Percepciones de alumnos de Física y Química de Educación Secundaria Obligatoria y Bachillerato sobre Wikipedia. Revista Eureka sobre Enseñanza y Divulgación de las Ciencias, 13 (1), 67-83. Recuperado de: https://bit.ly/2rCW4tJ

Viñals, A. (2016): El ocio conectado, móvil, transmedia y multisoporte de los jóvenes en la era digital. Journal of Communication, 13, 99-113. https://doi.org/10.14201/fjc20161399113 